ROMA1-TH/1397-04

SACLAY-T05/08

\title{
On Power and Complementarity of the Experimental Constraints on Seesaw Models
}

\author{
Isabella Masina $^{a)}$ and Carlos A. Savoy ${ }^{b)}$ \\ a) Centro Studi e Ricerche "E. Fermi", Rome, Italy, and \\ INFN, Sezione di Roma, Rome, Italy \\ b) Service de Physique Théorique, CEA - Saclay, France*
}

\begin{abstract}
We demonstrate the impact that present lepton flavour and CP violation data neutrino oscillations, baryon asymmetry of the universe, flavour violations in charged lepton decays and lepton electric dipole moments - have on supersymmetric seesaw theories by analysing the class of models based on a $U(1)$ flavour symmetry. The fermion $U(1)$ charges are determined to a large extent by the data, the flavour patterns being naturally defined through their choice. The selected models generically predict $\mu \rightarrow e \gamma$ within the reach of the projected experiments, which could be sensitive enough to exclude the whole class of them. By now, the present sensitivity to $\mu \rightarrow e \gamma$ already provides stringent bounds on the contribution of the seesaw couplings to the lepton electric dipole moments.
\end{abstract}

*Laboratoire de la Direction des Sciences de la Matière du Commissariat à l'Énergie Atomique et Unité de Recherche Associée au CNRS (URA 2306). 


\section{Introduction}

The simplest way to provide for light neutrino masses is the seesaw mechanism 1]. However, even in its basic type I version, the flavour pattern of the Dirac couplings $Y_{\nu}$ and the Majorana mass matrix $M_{R}$ remains largely undetermined. Indeed, the already very accurate data on the atmospheric and solar mass differences and mixing angles cannot discriminate between effective neutrino mass matrices, $m_{\nu}$, with different overall patterns. The potential measurements of other parameters in $m_{\nu}$ cannot resolve the indeterminacy which is an intrinsic feature of the heavy neutrino decoupling process - see e.g. [2]-6]. Complementary informations on the $Y_{\nu}$ and $M_{R}$ flavour patterns are to be found in the quantum effects involving the heavy neutrinos. It is well known that this is the case of the lepton asymmetry of the universe [7] and, assuming supersymmetry, of charged lepton dipole transitions, like the lepton flavour violating (LFV) decays $\ell_{i} \rightarrow \ell_{j} \gamma[8]$ and the CP violating (CPV) lepton electric dipole moments $(\mathrm{EDM}) d_{i}$ [9, 10. Without supersymmetry the large LFV discovered in neutrino oscillations and the CPV needed for leptogenesis do not lead to any measurable effect in the charged lepton interactions [11], in spite of the high sensitivity reached, in particular, in $\mu \rightarrow e \gamma$ [12] and $d_{e}$ [13]. Anyhow, protecting the Fermi scale with respect to the large mass scales involved in seesaw models requires a suitable mechanism - such as the supersymmetry framework, which is adopted in this work - and the corresponding new physics at scales not far from the $\mathrm{TeV}$ region. This is basically the region that lepton experiments are already testing for LFV and CPV sources.

The predictions for leptogenesis and charged lepton dipole transitions have very different dependences on $Y_{\nu}$ and $M_{R}$ with respect to neutrino oscillations, yet the extraction of the constraints on the seesaw parameters is not straightforward. Firstly, there are more parameters than experimental constraints; secondly, their analysis relies on additional assumptions, e.g., the neutrino initial abundance and the supersymmetric masses. Therefore one is forced to reduce the number of parameters by considering a model or a class of models at a time. Of course, the interest of these analyses depends on the naturalness of the assumptions and the robustness of the results with respect to variations in the parameters.

In this paper, we contribute to this exploratory work and show the power and the complementarity of the combined constraints from all these experiments by studying a popular class of models: the supersymmetric Froggatt-Nielsen ones where the seesaw parameters are restricted by a $U(1)_{F}$ flavour symmetry 14. Actually, these models [15, 16, 17] seem to best balance theoretical simplicity and consistency with experiments ${ }^{1}$. Their basic ingredients are: the charges of the three lepton doublets, $\ell_{i}(i=1,2,3)$, and those of the three heavy right handed neutrinos, $n_{i}$; one small parameter, $\epsilon$, associated to the breaking of $U(1)_{F}$ and to a charge -1 . The abelian flavour symmetry fixes the matrices $Y_{\nu}$ and $M_{R}$ up to $O(1)$ coefficients and thus

\footnotetext{
${ }^{1}$ See e.g. [18] for a comparison with less or more structured $U(1)_{F}$ models.
} 
the $\epsilon$ dependence of the various lepton observables in terms of the charges $\ell_{i}$ and $n_{i}$. This class of models has at least seven attractive features : (i) there is only one small parameter $\epsilon$ besides the $B-L$ breaking scale $v_{B-L}$; (ii) the hierarchies in $M_{R}$ depend only on $n_{i}$; (iii) the ratio between the (non-vanishing) $Y_{\nu}$ couplings of any right handed neutrino only depends on corresponding $\ell_{i}$ differences, so , (iv) $m_{\nu}$ has hierarchical eigenvalues depending only on $\ell_{i}$ differences; $(v)$ the models are natural and the hierarchies do not rely on the unknown $O(1)$ coefficients; (vi) the texture (the zero entries) of $Y_{\nu}$ is determined by supersymmetry (analytic superpotential); (vii) the hierarchies in the $Y_{\nu}$ elements are fixed by the $U(1)_{F}$ invariance of the superpotential. It has one (well known) slightly unattractive feature: some amount of tuning is needed to preserve the hierarchy between the solar and the atmospheric mass differences.

Of course, many aspects of these models have been studied in the literature, mostly at a time when the experimental situation of neutrino oscillations was more poorly established, leaving more freedom for the parameters $\ell_{i}$ and $\epsilon$. One of the first studies on leptogenesis considered models of this kind and showed that the final lepton asymmetry does not depend on $M_{2}$ and $M_{3}$ but only on the lightest eigenvalue, $M_{1}$, of $M_{R}$ [19]. LFV has been considered in some models of this class, and it was noticed that with $O(1)$ Yukawa couplings in $Y_{\nu}$ the predictions are close to the experimental limits for $\mu \rightarrow e \gamma$ [20]-23]. The significant improvement in our knowledge of neutrino oscillation parameters motivates our reappraisal of the abelian flavour symmetry models.

We show that, in this framework, the mass eigenvalues $M_{i}$ of $M_{R}$ are already almost fixed by present experiments and basically the whole class of models might be excluded by future ones [24]. In addition, we also derive bounds on the lepton EDM from the limits on $\mu \rightarrow e \gamma$ (which do not apply to other seesaw models) to conclude that the phases in $Y_{\nu}$ alone are not enough to radiatively induce lepton EDM even at the level of the planned experiments [25, 26].

We first consider models where, from the choice of the $U(1)_{F}$ charges, no texture zeros are natural in $Y_{\nu}$, in the sense that they are not required by supersymmetry analyticity. A characteristic of this whole class of models is that, in the seesaw formula, $m_{\text {atm }}$ gets roughly equivalent contributions from all heavy neutrinos. This fact by itself puts an upper bound $O\left(5 \times 10^{14}\right) \mathrm{GeV}$ on all the $M_{i}$. Oscillation experiments then ask for $\ell_{2}=\ell_{3}=\ell_{1}-1$ and $\epsilon=O\left(m_{\text {sol }} / m_{\text {atm }}\right.$ ) (actually close to the Cabibbo angle, often chosen for $\epsilon$ in models for the quark mass hierarchies). The models then yield the prediction $U_{e 3}=O\left(m_{\text {sol }} / m_{\text {atm }}\right)$, to be tested in the near future. The heavy neutrino charges, $n_{i}$ remain free and so do the $M_{i}$.

The next step is to show that the interplay between the constraints from leptogenesis, LFV and EDM, allow to gain significant information on the spectrum of right handed neutrinos in this class of models. With the $m_{\text {atm }}$ matrix elements fixed up to $O(1)$ coefficients, leptogenesis is possible in these models only if $M_{1}=O\left(10^{11}\right) \mathrm{GeV}$. 
The rate of LFV depends linearly on $M_{3}$, while EDM depend on the product $M_{2} M_{3}$. The limit on $M_{3}$ from the $\mu \rightarrow e \gamma$ search presently is two orders of magnitude above the leptogenesis value for $M_{1}$ and is on the way to be improved. Thus, this class of models has the nice feature that the comparison between theoretical parameters and the more restrictive experimental data is one-to-one in practice, giving also some predictions for quantities to be better measured in the future. As a matter of fact, EDM turn out to be so small that the only constraint on $M_{2}$ comes from its definition, $M_{1} \leq M_{2} \leq M_{3}$. This is because there is a relation between lepton EDM and LFV lepton decays while the latter are related by the $U(1)_{F}$ symmetry. Therefore all these quantities are bounded from the strong limits on $\mu \rightarrow e \gamma$, providing further tests of the models.

In this class of models, the strong upper limits on the heavy neutrino masses are related to the fact that their contributions to $m_{\text {atm }}$ are comparable. However, the heaviest neutrino can be naturally decoupled from the $\mu$ and $\tau$ doublets if one chooses $n_{3}$ low enough so that the corresponding matrix elements in $Y_{\nu}$ are zero by the analyticity of the supersymmetric Yukawa couplings. This alternative class of models is also studied here and we show that $M_{3}$ gets close to $O\left(M_{G U T}\right)$. However, the somewhat surprising result is that the would-be decoupled heavy neutrinos are even more coupled in $Y_{\nu}$ ! As a consequence these models are already generically excluded by the $\mu \rightarrow e \gamma$ data. Barring a complete decoupling of any heavy neutrino from all light ones is enough to select a very restricted, hence predictive, class of models when the experimental constraints are imposed.

A comment is in order on the supersymmetric flavour problem in the context of flavour theories, in particular those based on abelian groups. In general, the breaking of the $U(1)_{F}$ symmetry defining the small number $\epsilon$ induces supersymmetry breaking components for both $D$ and $F$ auxiliary fields along $U(1)_{F}$ charged directions, which in turn generically generate soft terms with LFV misalignment and CPV phases [27]. The flavour non-diagonal contributions to the soft slepton masses come out of order $\left(\ell_{j}-\ell_{i}\right) \epsilon^{\ell_{j}-\ell_{i}}$ and $\left(n_{j}-n_{i}\right) \epsilon^{n_{j}-n_{i}}$ with respect to the diagonal slepton masses ${ }^{2}$. They are analogous (up to charge difference factors) to the pattern of the radiative corrections discussed in this paper, since their $\epsilon$ dependence is basically fixed by the $U(1)_{F}$ broken symmetry too. A fine-tuning between the mostly constrained tree-level and radiative contributions looks very unnatural. Hence, one can naturally assume that each contribution is separetely restricted and should be separetely cared for. In particular, a suppression mechanism for both the $D$ and $F$ supersymmetry breaking associated to the $U(1)_{F}$ symmetry breaking, would lessen the tree-level contributions without any impact on the radiative ones.

\footnotetext{
${ }^{2}$ The origin of these flavour problems is related to the structure of the Kahler metric and the Yukawa interactions discussed in Appendix B. When the $U(1)_{F}$ is anomalous, its breaking is induced by a Fayet-Iliopoulos term and the associated $D$-terms give rise to an inversed hierarchy for the scalar masses and potentially large LFV and CPV in the mass matrices in the charged lepton flavour basis [27.
} 
The paper is organised as follows. The models are defined in section 2. The resulting effective neutrino mass matrix is confronted to the oscillation data constraints in section 3 and the general structure of $m_{\nu}$ is obtained. In section 4 , the leptogenesis requirements are imposed on the selected models to obtain the order of magnitude of $M_{1}$. The seesaw induced LFV effects in supersymmetric models are briefly reviewed in section 5 , with the limits from the $\operatorname{BR}(\mu \rightarrow e \gamma)$ upper bound given as functions of the slepton and gaugino masses. In the case of the models without holomorphic zeros in $Y_{\nu}$, one gets upper limits on $M_{3}$ which are quite low and will be further lowered by the future $\mu \rightarrow e \gamma$ measurements. Section 6 starts with a recall of the general seesaw induced EDM formulae written so to make their connection to the LFV transitions manifest. Some details are given in appendix A. Then, they are applied to the models discussed here where the indirect limits on $d_{e}$ from $\mu \rightarrow e \gamma$ supersede those from the $d_{e}$ experimental searches. In section 7 , models with naturallly vanishing couplings in $Y_{\nu}$ are considered and analysed along the same lines as in the previous sections. Some details are given in appendix B.

\section{The model}

The model is defined in the lepton sector by the following set of $U(1)_{F}$ charges: $\ell_{i}, e_{i}$ and $n_{i},(i=1,2,3)$ for the lepton electroweak doublets, charged and neutral singlets, respectively; $h_{u}, h_{d}$ for the electroweak symmetry breaking Higgs fields with v.e.v.'s $v_{u}, v_{d}$, respectively; $2 q$ for the field(s) breaking lepton number by two units in the Majorana masses and associated with the scale $v_{B-L}$. The $U(1)_{F}$ symmetry breaking field charge is normalized to -1 and the small flavour symmetry breaking parameter, $\epsilon$, is provided by the ratio of its v.e.v. and the cut-off of the flavour theory.

We first concentrate on the class of models such that all entries in the leptonic mass matrices are non-zero in the $U(1)_{F}$ basis, as any charge imbalance in the corresponding terms in the effective superpotential can be compensated by analyticitypreserving insertions of $\epsilon$. Hence, the flavour structure of the matrices in the leptonic sector is determined, up to $O(1)$ coefficients, by the following non-negative powers of $\epsilon$ :

$$
\left(Y_{\ell}\right)_{i j}=g_{i j} \epsilon^{e_{i}+\ell_{j}+h_{d}}, \quad\left(Y_{\nu}\right)_{i j}=h_{i j} \epsilon^{n_{i}+\ell_{j}+h_{u}}, \quad\left(M_{R}\right)_{i j}=r_{i j} \epsilon^{n_{i}+n_{j}+2 q} v_{B-L}
$$

where all the elements of the matrices $g, h$ and $r$ are $O(1)$. The effective neutrino mass matrix results in the expression

$$
\left(m_{\nu}\right)_{i j}=\left(Y_{\nu}^{T} M_{R}^{-1} Y_{\nu}\right)_{i j} v_{u}^{2}=\left(h^{T} r^{-1} h\right)_{i j} \epsilon^{\ell_{i}+\ell_{j}+2 h_{u}-2 q} \frac{v_{u}^{2}}{v_{B-L}} .
$$


After studying these models in detail we shall extend our analysis to models where some powers in (II) are negative and the corresponding entry vanishes in the $U(1)_{F}$ basis.

Models with non-zero entries have several interesting properties for the purposes of our analysis. Firstly, integrating out the Froggatt-Nielsen field preserves the analyticity in the effective theory, at least at the lowest order in $\epsilon$ and the powers of $\epsilon$ are given by the charge imbalance in the effective operators. This is evident in (2), where $m_{\nu}$ is independent of the charges $n_{i}$ [15] and gives no information on the heavy right handed neutrino spectrum. Secondly, the structure - in terms of powers of $\epsilon$ - of the unitary transformations diagonalizing the Yukawa couplings and the Majorana mass matrix in (11) is fixed by the differences between the $U(1)$ charges of different generations. As a consequence, in the basis where $Y_{\ell}$ and $M_{R}$ are diagonal, only the elements of $g, r$ (now diagonal) and $h$ change with respect to eq. (11), but they generically remain $O(1)$ (for a detailed proof see e.g. 19]). Since the abelian flavour symmetry only fixes the powers of $\epsilon$ up to arbitrary $O(1)$ coefficients, it is convenient and generically equivalent to work directly in the basis where $Y_{\ell}$ and $M_{R}$ are diagonal. Hence, in the following we choose this basis while keeping the same notations as above for simplicity. In particular (2) is now in the physical charged lepton basis used in the fits of neutrino oscillations, also convenient to study charged LFV and CPV transitions, of course.

The heavy right handed neutrinos possess a characteristic property in this class of models: the branch ratios for their decays into each light lepton flavour are all of the same order of magnitude and proportional to $\epsilon^{2 \ell_{j}}$. This property is partially lost when some matrix elements in (11) vanish by analiticity as discussed in section 7.

\section{Neutrino Oscillations}

Let us start with the comparison of the effective neutrino mass matrix in (2) and the corresponding parameters as determined by the experiments. In our analysis we adopt the $3-\sigma$ ranges of ref. [28]:

$$
\begin{gathered}
m_{@}=\sqrt{m_{3}^{2}-m_{2}^{2}}=(.05 \pm .01) \mathrm{eV} \quad, \quad m_{\odot}=\sqrt{m_{2}^{2}-m_{1}^{2}}=(.0091 \pm .0005) \mathrm{eV} \\
.71 \leq \tan \theta_{23} \leq 1.45 \quad, \quad \tan \theta_{12}=.65 \pm .12 \quad, \quad \tan \theta_{13} \leq .21
\end{gathered}
$$

Of course, this comparison is present in several papers, e.g. [15, 16, 17, but the recent improvements in the experimental determination of neutrino oscillation parameters - in particular in the solar sector - now allow for a basically unique solution, that we turn to discuss. 
First of all, the very large atmospheric mixing angle requires $\ell_{2}=\ell_{3}=\ell$. It is convenient to introduce the parameter $\rho=\Delta_{23} / m_{3}^{2}$, where $\Delta_{23}$ is the sub-derminant of the $i, j=2,3$ sub-matrix of $m_{\nu}$ and $m_{3}$ is its largest eigenvalue. The bound on

$$
\sin \theta_{13} \approx \epsilon^{\ell_{1}-\ell} \frac{\rho+1}{\sqrt{2}} \frac{\left|\left(h^{T} r^{-1} h\right)_{12}+\left(h^{T} r^{-1} h\right)_{13}\right|}{\left|\left(h^{T} r^{-1} h\right)_{22}+\left(h^{T} r^{-1} h\right)_{33}\right|}
$$

requires $\ell_{1}>\ell$ leaving the larger elements in the $i, j=2,3$ sub-matrix of eq. (2) to be of $O\left(m_{@}\right)$. We can take $\ell_{1}=\ell+1$ up to a trivial re-scaling of $\epsilon$. From these two requirements, the flavour structure of $m_{\nu}$ in this class of models is fixed :

$$
\left(m_{\nu}\right)_{i j}=\left(h^{T} r^{-1} h\right)_{i j} \epsilon^{\delta_{i 1}+\delta_{j 1}} \epsilon^{2\left(\ell+h_{u}-q\right)} \frac{v_{u}^{2}}{v_{B-L}}
$$

This structure has also to accomodate the ratio $m_{\odot} / m_{@}=0.18 \pm 0.05$ as well as the relatively large but not maximal value of $\tan \theta_{12}$. One has $\rho \approx \cos ^{2} \theta_{12} m_{2} / m_{3}$ and $\tan \theta_{12}=O(\epsilon / \rho)$. Then, for $m_{@} \approx m_{3}$ and $m_{\odot} \approx m_{2}$, by using the experimental range for the solar angle one obtains $O(\epsilon)=m_{\odot} \sin 2 \theta_{12} / 2 m_{@} \approx .08 \pm .01$. In the spirit of the whole approach, we take advantage of the $O(1)$ arbitrariness in the matrices $h$ and $r$ to fix $\epsilon$ at the central value of $m_{\odot} / m_{@}, \epsilon=0.18$, from now on. From (4), it follows that $U_{e 3}=O(\epsilon)$, not so far from the present upper bound [17, 18].

Clearly, $\rho=O(\epsilon)$ requires a relatively mild tuning of the $O(1)$ coefficients, inherent to this class of models [15], which remains a weak point of the present class of models ${ }^{3}$. To illustrate it, in fig. 1) the $\rho$ distribution is shown for randomly generated $O(1)$ complex matrix elements in $h$ and $r$ and also for the small subset of the generated models that fulfil all the experimental constraints (3) - the latter magnified by a factor of 10 in the figure. The experimentally allowed region of $\cos ^{2} \theta_{12} m_{\odot} / m_{@}$ is also shown for comparison. One can estimate from the figure the probability for the generated models to satisfy the required tuning in $\rho$. Of course, the relatively high precision of the neutrino oscillation data (3) further reduces the experimentally viable models to the small fraction shown in the figure.

The heavy Majorana neutrinos all contribute the same order of magnitude to each element of $m_{\nu}$ independently of their masses, $M_{i}$. However, there is an upper bound on their masses if their couplings $Y_{\nu}$ are to remain perturbative. Indeed, from eq. (11) one has

$$
\epsilon^{2\left(\ell+h_{u}+n_{i}\right)} r_{i} \frac{v_{u}^{2}}{M_{i}}=\epsilon^{2\left(\ell+h_{u}-q\right)} \frac{v_{u}^{2}}{v_{B-L}}=\frac{m_{@}}{d_{@}} \quad, \quad \forall i
$$

\footnotetext{
${ }^{3}$ Models with single right handed neutrino dominance, where the suppression of $\rho$ becomes natural, were specifically proposed in order to overcome this problem 29].
} 


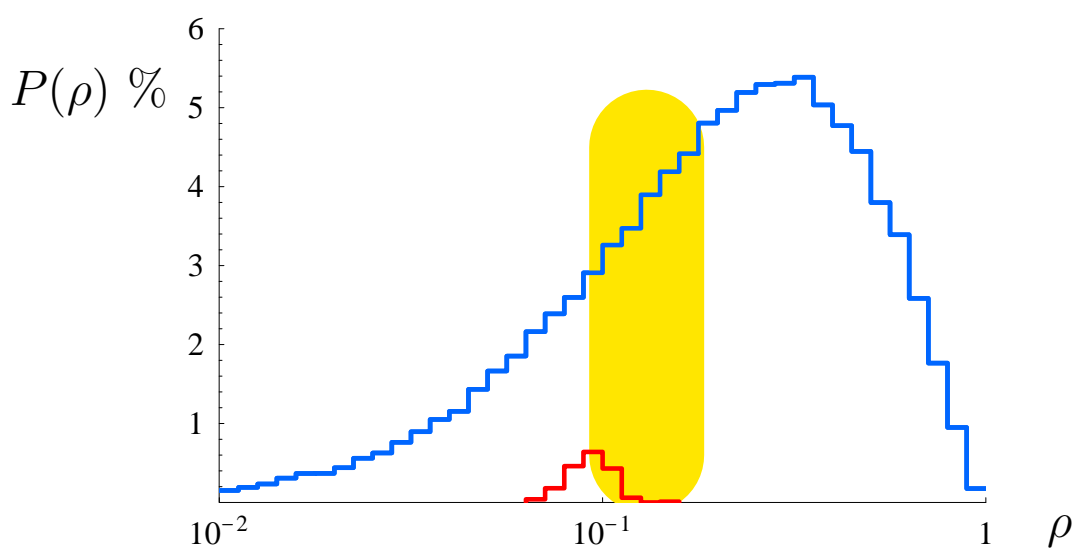

Figure 1: Distribution of $\rho$ obtained from a sample of models with charges $\ell_{1}-1=$ $\ell_{2}=\ell_{3} \equiv \ell, \epsilon=0.18$ and matrix elements $r_{i}, h_{i j}$ randomly generated among the complex numbers with absolute values between $0.3-3$. The upper (blue) distribution refers to the whole sample, while the lower (red) distribution is obtained by keeping only the models in the sample satisfying all the present neutrino oscillation bounds (3). The latter has been magnified by a factor of 10 for graphical reasons. The (yellow) shaded region represents the present range of $\cos ^{2} \theta_{12} m_{\odot} / m_{@}$.

where $d_{@}=\mathrm{O}(1)$ is the analog of $m_{@}$ for the dimensionless matrix $\left(h^{T} r^{-1} h\right)_{i j} \epsilon^{\delta_{i 1}+\delta_{j 1}}$. Since by assumption $\ell+h_{2}+n_{i} \geq 0$, we obtain the upper bound for each $M_{i}$

$$
\frac{M_{i}}{M_{@}}=\epsilon^{2\left(\ell+h_{u}+n_{i}\right)} d_{@} r_{i} \lesssim O(1) \quad, \quad M_{@}=\frac{v_{u}^{2}}{m_{@}} \simeq 5 \times 10^{14} \mathrm{GeV} \quad .
$$

Another characteristic property following from $\ell_{1}=\ell+1$, relevant for the predictions of the next sections, is the fact that all the right-handed neutrinos, $\nu_{i}^{c}$, couple to the first family doublet $\ell_{1}$ by a factor of $\epsilon$ less than to the other two family doublets. This means that $e, \nu_{e}$ are expected to be less important for leptogenesis (the decays into $\mu, \nu_{\mu}$ and $\tau, \nu_{\tau}$ are dominant) and also that LFV and CPV transition amplitudes involving the electron are roughly reduced by corresponding factors of $\epsilon=m_{\odot} / m_{@}$.

We now turn to discuss how other experimental data can give informations on the flavour structure of right handed neutrinos, starting with the constraints from the assumption of leptogenesis. 


\section{Leptogenesis}

One of the very first explicit calculations of leptogenesis with the seesaw [19] mechanism adopted models of the same class discussed here. It found that the lepton asymmetry critically depends on $M_{1}$ and is quite independent of the right handed neutrino mass hierarchy. Of course, the neutrino oscillation parameters were less constrained at that time. Here, we update and slightly generalize it and we explicitely find the favoured range of $M_{1}$, which is used in the next sections.

In the notations of ref. [30], adapted to the supersymmetric case [31, 32, 33], the resulting expression for the baryon asymmetry of the universe is:

$$
\begin{gathered}
\eta_{B}=-10^{-2} \varepsilon_{1} \kappa_{f}, \quad \varepsilon_{1}=\frac{1}{8 \pi} \sum_{j \neq 1} \frac{\operatorname{Im}\left[\left(Y_{\nu} Y_{\nu}^{\dagger}\right)_{j 1}^{2}\right]}{\left(Y_{\nu} Y_{\nu}^{\dagger}\right)_{11}} g\left(\frac{M_{j}^{2}}{M_{1}^{2}}\right), \\
g(x)=-\sqrt{x}\left[\frac{2}{x-1}+\ln \frac{1+x}{x}\right] \stackrel{x>>1}{\longrightarrow}-\frac{3}{\sqrt{x}}
\end{gathered}
$$

where $\kappa_{f}$ is the efficiency factor. In the present class of models we can take advantage of the $x>>1$ limit because both conditions that could invalidate this approximation are unnatural. Indeed, without fine-tuning, $M_{1}$ and $M_{2}$ cannot be degenerate at the level required for a resonant enhancement [34, and $M_{2}$ and $M_{3}$ cannot be a pseudoDirac pair [35]. In the strong washout regime, where the effective neutrino mass $\tilde{m}_{1}=\left(Y_{\nu} Y_{\nu}^{\dagger}\right)_{11} v_{u}^{2} / M_{1}$ is larger than the equilibrium neutrino mass $m_{*} \approx 0.8 \times$ $10^{-3} \mathrm{eV}$ [33], the dependence of $\kappa_{f}$ on the initial abundance is very small and well approximated by the power law [30, 32, 33]: $\kappa_{f} \approx(1.5 \pm 0.7) \times 10^{-4} \mathrm{eV} / \tilde{m}_{1}$. Thus, successful leptogenesis yields a general lower limit on $M_{1}$ as a function of $\tilde{m}_{1}$, as displayed, e.g., in ref. [30, 33.

In the models considered here, defining the $O(1)$ matrix $H_{i j}=\sum_{k} h_{i k} h_{j k}^{*} \epsilon^{2 \delta_{k 1}}$, one gets:

$$
\varepsilon_{1}=\frac{3}{8 \pi} \frac{M_{1} m_{@}}{v_{u}^{2}} \sum_{j \neq 1} \frac{\operatorname{Im}\left[H_{j 1}^{2}\right]}{H_{11} r_{j} d_{@}}=\frac{3}{8 \pi} O\left(\frac{M_{1}}{M_{@}}\right), \quad \tilde{m}_{1}=m_{@} \frac{H_{11}}{r_{1} d_{@}}=O\left(m_{@}\right) .
$$

Hence the approximation for $\kappa_{f}$ in the strong washout regime is valid and one gets for the baryon asymmetry of the universe:

$$
\eta_{B} \approx \frac{2 \pm 1}{2} \frac{10^{-7} \mathrm{eV}}{m_{@}} \frac{M_{1}}{M_{@}} \sum_{j \neq 1} \frac{\operatorname{Im}\left[H_{j 1}^{2}\right]}{H_{11}^{2}} \frac{r_{1}}{r_{j}}
$$




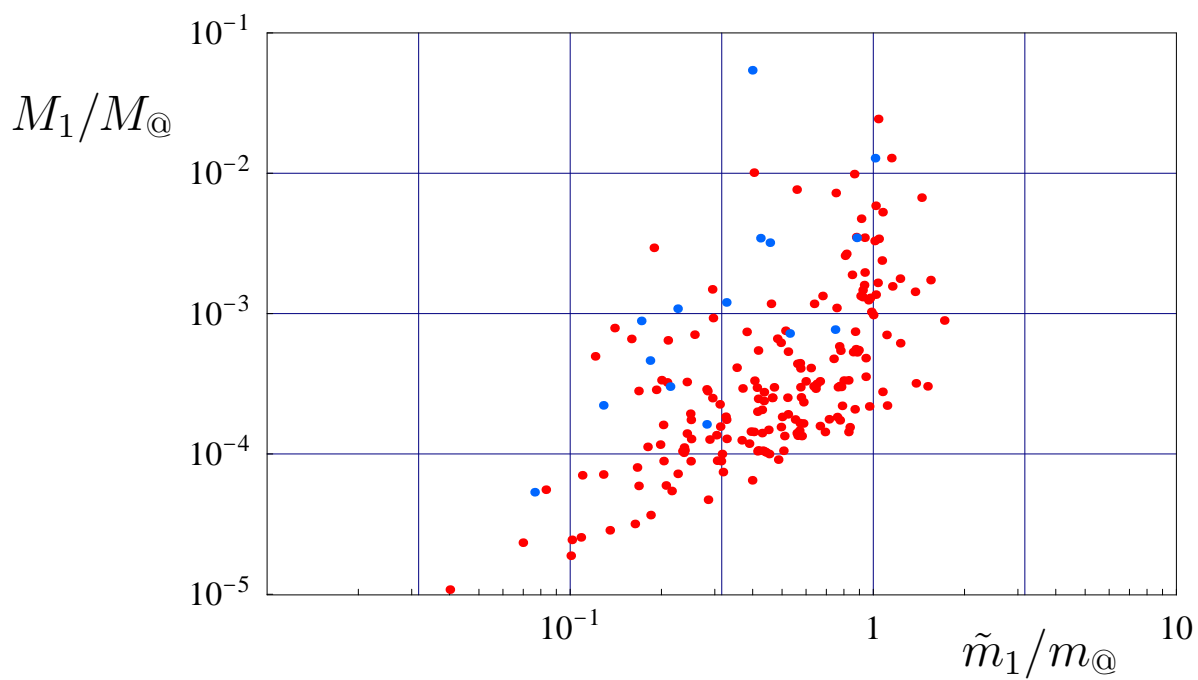

Figure 2: Scatter plot in the plane $M_{1} / M_{@}$, eq. (10), and $\tilde{m}_{1} / m_{@}$, eq. (9), for the models (described in the previous section) satisfying all the neutrino oscillation experimental data. The value of $M_{1} / M_{@}$ brings a theoretical uncertainty of a factor $1 / 2-2$. For the points in red the sine of the leptogenesis phase is $\geq 0.1$ while for those in blue it is $<0.1$.

to be compared to the experimental value $\eta_{B}=(6.3 \pm 0.3) \times 10^{-10}[36$. Therefore one derives, within the uncertainty of a factor $1 / 2-2$, the value of $M_{1} / M_{@}$ required by leptogenesis. This is shown in fig. 2 for the randomly generated models selected by the neutrino oscillation data as discussed in section 3 . The results favour $M_{1}=$ $O\left(10^{11} \mathrm{GeV}\right)$. It is well known that leptogenesis at such scale hides a potential gravitino problem which is solved if the gravitino is either stable 37] or heavier than $O(2 \mathrm{TeV})$ [38. Notice that, since $\varepsilon_{1}$ in eq. (9) turns out to be close to the DI upper bound [39], the above prediction for $M_{1}$ is actually close to its general lower limit for $\tilde{m}_{1}=O\left(m_{@}\right)$ [30].

Notice also that in $H_{j 1}$ the electron term is reduced by $\epsilon^{2}$ as compared to the $\mu$ and $\tau$ ones, so that the leptogenesis phase should mostly result from the latter.

\section{$5 \quad$ LFV}

We now turn to the experimental data that can provide informations on the heaviest right handed neutrino, namely, the LFV decays of the charged leptons. In a theory based on broken supersymmetry, $\ell_{i} \rightarrow \ell_{j} \gamma$ arise from the possible misalignment between the slepton and the charged lepton mass matrices. The expansion of the transition amplitude in terms of the relative misalignment of the slepton masses - or insertion approximation - is justified by the fact that these LFV rates are expected 
to be relatively small, as attested by the bounds on $\mu \rightarrow e \gamma$. There is a rich literature on the experimental constraints on this misalignment that emphasize the importance of the supersymmetric flavour problem in the lepton sector 40, 41]. Here, we make use of the results in 42 .

In supersymmetric theories the radiative corrections are wave function renormalisations by hermitean matrices, whose flavour and CP structures depend on the Yukawa couplings. In the context of the supersymmetric seesaw models, the radiatively induced misalignment was emphasized a long time ago [8] and more recent analyses [2, 20, 21, 23, 43, 44] were prompted by the determination of the neutrino oscillation parameters. Starting from CP and flavour conserving soft terms and taking $M_{R}$ and $Y_{\ell}$ real and diagonal at $M_{\mathrm{Pl}}$, the flavour and $\mathrm{CP}$ violations in slepton masses are introduced in the RG equations only by heavy neutrino $\nu_{k}^{c}$ loops through their flavour changing couplings $Y_{\nu i k}^{\dagger} Y_{\nu k j}, i \neq j$, until they successively decouple at $M_{k}$ yielding corresponding factors $\ln \left(M_{\mathrm{Pl}} / M_{k}\right)$. Since $Y_{\nu}$ couples $\nu_{k}^{c}$ to the lepton doublets, the seesaw radiative corrections mainly affect the doublet slepton masses $m_{L i j}^{2}$. Assuming universality in soft masses at $M_{P l}$, the misalignment in $m_{L}^{2}$ at the lowest order in the couplings $Y_{\nu}$ (defined at $\left.M_{P l}\right)$ is [ $]$ :

$$
\begin{aligned}
\delta_{i j}^{L L} & =\frac{m_{L i j}^{2}}{\bar{m}_{L}^{2}}=-\frac{1}{(4 \pi)^{2}} \frac{6 m_{0}^{2}+2 a_{0}^{2}}{\bar{m}_{L}^{2}} C_{i j} \\
C_{i j} & =\sum_{k} C_{i j}^{k}=\sum_{k} Y_{\nu k i}^{*} Y_{\nu k j} \ln \frac{M_{\mathrm{Pl}}}{M_{k}}
\end{aligned}
$$

where $m_{0}$ and $a_{0}$ are the universal soft breaking bilinear and trilinear terms at $M_{P l}$, respectively, and $\bar{m}_{L}$ is the average doublet slepton mass. We have isolated the contribution from each $\nu_{k}^{c}$ state, $C_{i j}^{k}$, for later convenience.

In the basis where $Y_{\ell}$ and $M_{R}$ are diagonal, the mass insertion approximation reads

$$
\mathrm{BR}\left(\ell_{i} \rightarrow \ell_{j} \gamma\right)=10^{-5} \mathrm{BR}\left(\ell_{i} \rightarrow \ell_{j} \bar{\nu}_{j} \nu_{i}\right) \frac{M_{W}^{4}}{\bar{m}_{L}^{4}} \tan ^{2} \beta\left|\delta_{i j}^{L L}\right|^{2} F_{\text {susy }}
$$

where $F_{\text {susy }}=O(1)$ is a function of supersymmetric masses which includes both the chargino and neutralino exchange (see e.g. [42, and references therein). With eq. (11), the resulting general limits on slepton misalignment 42 translate into limits on the $\left|C_{i j}\right|$ as functions of the supersymmetric masses and $\tan \beta\left[23\right.$, 4] ${ }^{4}$. In mSugra one can express the dependence on the susy spectrum in terms of $a_{0}, \tan \beta$ and two

\footnotetext{
${ }^{4}$ The figures in [23] only take into account the chargino contribution, not dominant in some regions of the supersymmetric masses [42. The neutralino contributions are included in the plots of ref. [4].
} 
masses: either the bino mass $\tilde{M}_{1}$ and the average singlet charged slepton mass $\bar{m}_{R}$, or the universal scalar and gaugino mass parameters, respectively $m_{0}$ and $M_{1 / 2}$. The upper limits on $\left|C_{\mu e}\right|$ from the present upper bound $\operatorname{BR}(\mu \rightarrow e \gamma)<10^{-11}$ are shown in fig. 3] for $\tan \beta=10$. The two choices for $a_{0}$ are meant to give an idea of the theoretical uncertainty: a) $a_{0}=0$ (dotted lines) and b) $a_{0}=m_{0}+M_{1 / 2}$ (solid lines). For different choices of $\tan \beta$ and/or better sensitivities than the present one, it is enough to rescale according to (12). The same plot can be used to estimate the upper bounds on $C_{\tau \mu}$ from $\tau \rightarrow \mu \gamma$ by taking into account the factor $\operatorname{BR}(\tau \rightarrow \mu \bar{\nu} \nu) \approx 17 \%$.

In spite of the much poorer determination of $\epsilon$ at the time, seesaw models of the present class were among the first analysed to get predictions for $\mu \rightarrow e \gamma$ and $\tau \rightarrow \mu \gamma$. It was pointed out that for models whose charges allow for $O(1)$ couplings in $Y_{\nu}$, the predictions for $\mu \rightarrow e \gamma$ are at the level of the present limits and the planned improvement [24] would test them [20, 21] (as can also be inferred from fig. 3). The relevance of $\tau \rightarrow \mu \gamma, \tau \rightarrow e \gamma$ was emphasized as well. Of course, such considerations surpass these particular models. A general discussion of LFV decays for the three possible classes of seesaw models, defined in terms of the dominance mechanism in the neutrino masses, was presented in ref. 23].

Actually, what characterizes the present class of models is that all $\nu^{c}$ 's contributions to $m_{\nu}$ are on an equal-footing, as expressed by eq. (6), so that

$$
\begin{aligned}
C_{i j}^{k} & =h_{k i}^{*} h_{k j} \epsilon^{\delta_{i 1}+\delta_{j 1}} \epsilon^{2\left(n_{k}+\ell+h_{u}\right)} \ln \frac{M_{\mathrm{Pl}}}{M_{k}} \\
& =\frac{h_{k i}^{*} h_{k j}}{r_{k} d_{@}} \epsilon^{\delta_{i 1}+\delta_{j 1}} \frac{M_{k}}{M_{@}} \ln \frac{M_{\mathrm{Pl}}}{M_{k}}, \quad \forall k .
\end{aligned}
$$

Therefore, from the experimental limits on $\left|C_{i j}\right|$ one gets directly bounds on the heaviest Majorana mass $M_{3}$ - unless, for $M_{3} \simeq M_{2}, C_{i j}^{3}$ and $C_{i j}^{2}$ strongly interfere, which amounts to some tuning of the free parameters. On the other hand, the leptogenesis constraint on the lightest mass $M_{1}$, provides a lower limit, $C_{i j} \gtrsim \epsilon^{\delta_{i 1}+\delta_{j 1}}\left(10^{-2}-10^{-3}\right)$, hence a lower bound on $\operatorname{BR}\left(\ell_{i} \rightarrow \ell_{j} \gamma\right)$.

We first discuss in some detail the bound on $M_{3}$ which follows from $\mu \rightarrow e \gamma$. The right panel of fig. 3 shows the scatter plot of $\left|C_{\mu e}^{3}\right|$ - eq. (13) - as a function of $M_{3} / M_{@}$ for the models fulfilling all neutrino oscillation and leptogenesis constraints (of course similar plots apply also to $k=1,2$ ). Since, with the value of $\epsilon$ fixed as in section 3, $\left|C_{\mu e}^{3}\right|=O\left(M_{3} / M_{@}\right)$, the experimental upper bounds on $\left|C_{\mu e}\right|$ and $M_{3} / M_{@}$ are of the same order of magnitude:

$$
\frac{M_{3}}{M_{@}} \lesssim O\left(\left|C_{\mu e}\right|^{\text {u.b. }}\right) .
$$

These upper bounds are displayed in the left plot of fig. 3. With the present experimental sensitivity, they already provide meaningful limits on $M_{3}$, in spite of 

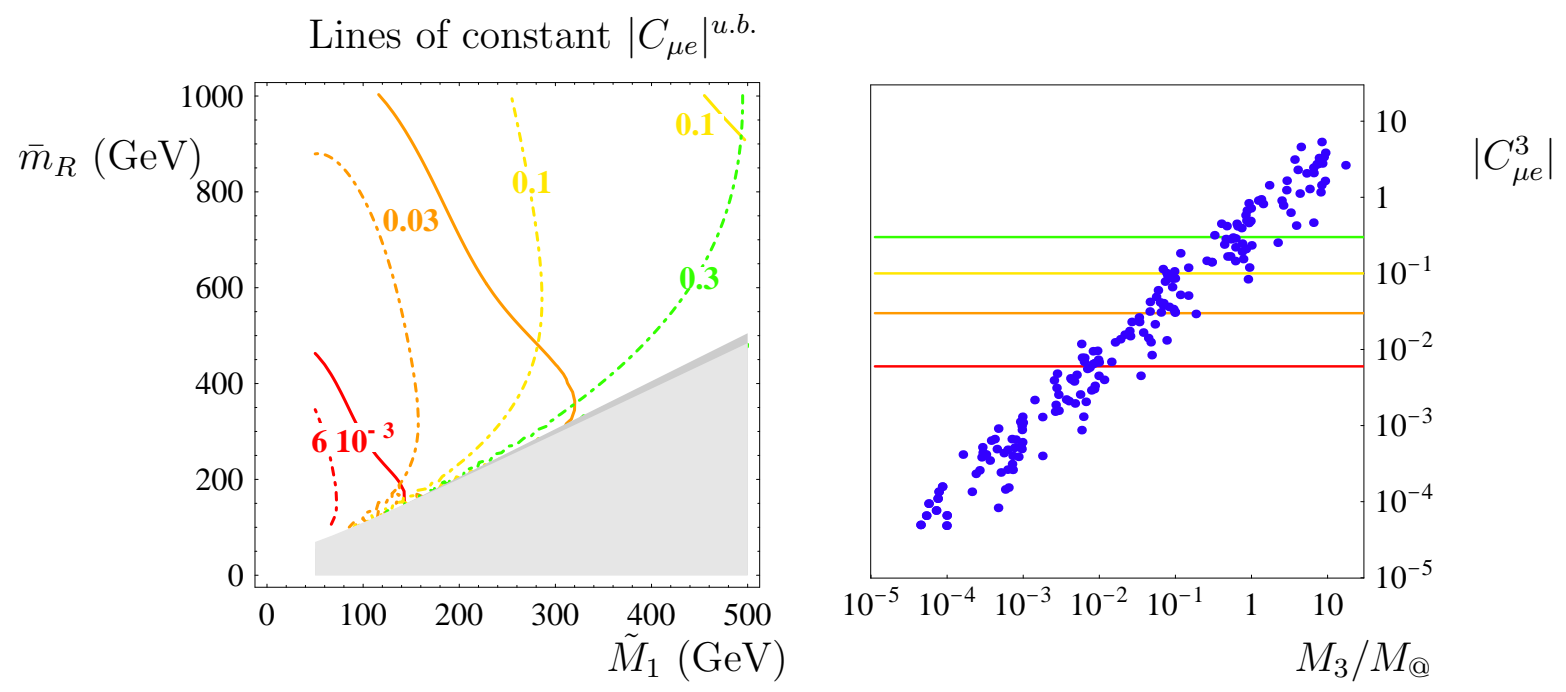

Figure 3: The left plot shows the upper bounds on $\left|C_{\mu e}\right|$ for $\operatorname{BR}(\mu \rightarrow e \gamma) \leq 10^{-11}$ and $\tan \beta=10$. The plot, adapted from that in [4, includes both the chargino and neutralino contributions and assumes mSugra with $a_{0}=0$ (dotted line) and $a_{0}=m_{0}+M_{1 / 2}$ (solid one). The right plot shows the scatter plot of $\left|C_{\mu e}^{3}\right|$, eq. (13), as a function of $M_{3} / M_{@}$ for the models fulfilling all neutrino oscillation and leptogenesis constraints, with $M_{1}<10^{11} \mathrm{GeV}$. We take $M_{3}$ to span its allowed range, namely $M_{1} \leq M_{3} \leq d_{@} r_{3}$.

the uncertainties in the supersymmetric spectrum. For instance, with $\tan \beta=10$, $M_{3} / M_{@} \lesssim 0.3(1)$ for the mass region below the orange (yellow, resp.) line, some two orders of magnitude below the gauge coupling unification scale! With the planned three orders of magnitude improvement in $\operatorname{BR}(\mu \rightarrow e \gamma)$, it would become $M_{3} / M_{@} \lesssim$ $10^{-2}$ in that mass regions, which is close to the $M_{1}$ range selected by leptogenesis, where $C_{\mu e} \gtrsim C_{\mu e}^{1} \approx\left(10^{-3}-10^{-4}\right)$. Therefore, for this class of models, there are fair perspectives to detect a positive signal in $\mu \rightarrow e \gamma$ searches. Alternatively, barring destructive interference among the $C_{\mu e}^{k}$, the future experiments would rule out this whole class of models in the region of the parameter space where $\left|C_{\mu e}\right|^{\text {u.b. }} \lesssim 10^{-4}$. For $\tan \beta \geq 20$, it actually coincides with the area below the red lines which also corresponds to a sizeable supersymmetric contribution to $(g-2)_{\mu}$ (see 42 and references therein). This clearly shows the power of the combined constraints from $\mu \rightarrow e \gamma$ and leptogenesis in this class of models.

As already noticed, the couplings to $e$ are reduced with respect to $\mu$ and $\tau$ by a factor $O(\epsilon)=O\left(m_{\odot} / m_{@}\right)$, so that $C_{\mu e}^{k}=O\left(C_{\tau e}^{k}\right)=O(\epsilon) C_{\tau \mu}^{k}$. This simple link to the $m_{\nu}$ pattern is a characteristic of the model. In particular, the model predicts: $\mathrm{BR}(\mu \rightarrow e \gamma): \operatorname{BR}(\tau \rightarrow \mu \gamma): \operatorname{BR}(\tau \rightarrow e \gamma)=\epsilon^{2}: .17: .17 \epsilon^{2}$, while the present experimental upper bounds are in the ratios $10^{-5}: 1: 1$, respectively. Hence, in this model, from the experimental limit on $\mu \rightarrow e \gamma$, the other two LFV transitions are 
predicted many orders of magnitude below their present experimental limits.

\section{Lepton EDM}

There is a rich literature about the constraints that lepton EDM searches put on the CPV pattern of the MSSM and its extensions [45, 46], in particular, on the slepton mass matrices. It is useful to separate them into two kinds: i) lepton flavour conserving (FC) parameters, namely, the phase of the $\mu$-term and phases in the flavour diagonal chirality flipping slepton masses, or A-terms; ii) phases in the lepton flavour violating (FV) elements of the slepton mass matrices. Very restrictive conditions on some of those phases arise from the present limits on $d_{e}$ which are to be considerably strengthened in the near future 25. The present limit on $d_{\mu}$ is not very significant but a new experiment 26] could bring relevant results. The first conclusion is that supersymmetric models must possess a mechanism to effectively inhibit CP phases in the soft terms at $M_{P l}$. The second one is that the radiative corrections must keep the induced CP phases in the soft terms below the experimental limits. In this section we concentrate on the constraints on the radiative corrections arising from the Yukawa couplings of the supersymmetric seesaw models.

The main contributions to $d_{i}$ 's of either kind depend on the slepton mass parameters as follows:

$$
d_{i}^{F C}=\frac{e^{3} \tilde{M}_{1} I_{B}}{32 \pi^{2}|\mu|^{2} \cos ^{2} \theta_{W}} m_{\ell_{i}} \operatorname{Im}\left(a_{i}\right) \quad d_{i}^{F V}=\frac{e^{3} \tilde{M}_{1} \mu \tan \beta I_{B}^{\prime \prime}}{32 \pi^{2}|\mu|^{2} \cos ^{2} \theta_{W}} \operatorname{Im}\left(\delta^{R R} m_{\ell} \delta^{L L}\right)_{i i}
$$

in the basis where $Y_{\ell}$ and $M_{R}$ are real and diagonal and with the FC $A$-terms written as $A_{i}=a_{i} Y_{\ell_{i}}$. The functions $I_{B}$ and $I_{B}^{\prime \prime}$ depend on the slepton, chargino and neutralino masses as defined in ref. [42]. The first contributions to $d_{i}^{F V}$ are second order in the insertion approximation. Only the FV elements of the (hermitian) matrices $\delta^{R R}, \delta^{L L}$, those responsible for LFV decays, contribute in (15). Hence $d_{e}^{F V}$ is linked to $\tau \rightarrow e \gamma$ and $\mu \rightarrow e \gamma, d_{\mu}^{F V}$ to $\tau \rightarrow \mu \gamma$ and $\mu \rightarrow e \gamma, d_{\tau}^{F V}$ to $\tau \rightarrow \mu \gamma$ and $\tau \rightarrow e \gamma$ (see, e.g., 42 for a general discussion). Here, these links are analysed anew in the framework of the supersymmetric seesaw mechanism as one source of LFV and CPV.

We start with real $\mu$ and no $\mathrm{CP}$ or lepton flavour violations in the $A$-terms at $M_{P l}$. In particular, $\operatorname{Im} a_{i}=0$. Then, $\mathrm{CP}$ violations are introduced through the RG running only by the heavy neutrino couplings $Y_{\nu}$. The decoupling of right handed neutrinos yields an effective MSSM, with neutrino masses and CP phases in the slepton masses. As discussed in section 5 , the hermitian matrices $C^{k}$ appear at one loop and the $\left|C_{i j}^{k}\right|$ are bounded by the $\ell_{i} \rightarrow \ell_{j} \gamma$ decays. However, since $C_{i i}^{k}$ is real, 
there is no CPV phase to induce $d_{i}$. This means that the radiatively induced CP violations start at the two-loop level and, in the leading log development, come from the phases of LFV elements in the products $\operatorname{Im}\left(\sum_{j} C_{i j}^{k} C_{j i}^{k^{\prime}}\right), k \neq k^{\prime}$. By obtaining the dependence of $d_{i}^{F C}$ and $d_{i}^{F V}$ on the $C_{i j}^{k}$ one makes more manifest how they are constrained by the LFV experiments. Let us first discuss these relations more generally, before turning back to the class of models analysed here.

\subsection{General link between EDM and LFV}

Recently, the dominant two-loop contributions to $\operatorname{Im} a_{i}$ and $\delta^{R R}, \delta^{L L}$ in the expressions of the supersymmetric seesaw radiatively induced EDMs have been calculated 9, 10, 47. Here, the results are rearranged to make clear the general connection between LFV and EDM, by expressing $d_{i}$ in terms of the $C_{i j}^{k}$. The original results in [10] and some detailed expressions are presented in App. A.

Assuming mSugra boundary conditions at $M_{P l}$ for simplicity, the result [9, 10] for $d_{i}^{F C}$ is obtained by replacing in (15):

$$
\operatorname{Im}\left(a_{i}\right)=\frac{8}{(4 \pi)^{4}} a_{0} \sum_{k>k^{\prime}} \frac{\ln \left(M_{k} / M_{k^{\prime}}\right)}{\ln \left(M_{P l} / M_{k^{\prime}}\right)} \operatorname{Im}\left(\sum_{j \neq i} C_{i j}^{k} C_{i j}^{* k^{\prime}}\right)
$$

Notice that since $\operatorname{Im}\left(\sum_{i} a_{i}\right)=0$ the FC contribution leads to the sum rule:

$$
\sum_{i} d_{i} / m_{i}=0
$$

To obtain $d_{i}^{F V}$, one has to replace in (15) the leading two-loop result [10, 47] for the double insertion term, which is:

$$
\begin{aligned}
\operatorname{Im}\left(\delta^{R R} m_{\ell} \delta^{L L}\right)_{i i} & =\frac{8}{(4 \pi)^{6}} \frac{\left(6 m_{0}^{2}+2 a_{0}^{2}\right)\left(6 m_{0}^{2}+3 a_{0}^{2}\right)}{\bar{m}_{L}^{2} \bar{m}_{R}^{2}} \frac{m_{\tau}^{2}}{m_{t}^{2}} m_{\ell_{i}} \tan ^{2} \beta I_{i}^{F V} \\
I_{i}^{F V} & =\sum_{k>k^{\prime}} \widetilde{\ln }_{k^{\prime}}^{k} \operatorname{Im}\left(\sum_{j \neq i} C_{i j}^{k} \frac{m_{\ell_{j}}^{2}}{m_{\tau}^{2}} C_{i j}^{k^{\prime *}}\right)
\end{aligned}
$$

where $\bar{m}_{R}$ and $\bar{m}_{L}$ are average masses of the sleptons of each chirality, and the logarithmic dependence on the masses $M_{k}$ represented by $\widetilde{\ln }_{k^{\prime}}^{k}$ is defined in App. A. The sum rule (17) is not valid as the FV contribution rather leads to the sum rule:

$$
\sum_{i} m_{i} d_{i}=0
$$


There are other three points to notice with respect to these expressions:

i) The EDM phase results from the (three different) relative phases between $C_{i j}^{k}$ and $C_{i j}^{k^{\prime}}$, namely between the $\nu_{k}^{c}$ and $\nu_{k^{\prime}}^{c}$ contributions to $\ell_{i} \rightarrow \ell_{j} \gamma$.

ii) The phase in the quark CKM matrix is large and there is no known reason to think of smaller phases in the lepton sector, thus one expects the EDM phases to be generically large.

iii) As already discussed, $\left|C_{i j}^{k}\right|$ are constrained by the experimental limits on the $\mathrm{LFV}$ decays $\ell_{i} \rightarrow \ell_{j} \gamma$. However, at present the only significant bound from LFV decays is that on $\left|C_{\mu e}\right|$ from $\mu \rightarrow e \gamma$.

It has been shown in ref. [10 that with (16) and (18), $d_{i}^{F V}>d_{i}^{F C}$ for $\tan \beta \geq 10$. Indeed, the FC contribution vanishes for $a_{0}=0$, while for $a_{0} \sim m_{0}$, as $d_{i}^{F V} / d_{i}^{F C} \propto$ $(\tan \beta)^{3}$, the FV contribution dominates over the FC one for large $\tan \beta$ in spite of their different sparticle mass dependence. We refer to [10] for general analytical and numerical studies of these seesaw mechanism as a source of lepton EDM.

For those reasons, we mainly concentrate on $d_{i}^{F V}$. In fig. 4, the limit on $I_{e}^{F V}$ obtained from the bound $d_{e} \lesssim 10^{-32} \mathrm{e} \mathrm{cm}$ is shown for $\tan \beta=10$ and $a_{0}=0$ (dotted lines) or $a_{0}=m_{0}+M_{1 / 2}$ (solid ones). With the present bound the limits are $10^{5}$ larger and quite useless. Indeed, if the Yukawa couplings are at most $O(1)$ and the masses $M_{k}$ in the range selected by neutrino masses and leptogenesis, one has $I_{i}^{F V}<O\left(10^{-3}\right)$. Then, only for light sparticles and large $\tan \beta$ the present experimental bound on $d_{e}$ constrains $I_{e}^{F V}$ to be below this upper bound [10].

Finally, from the explicit expression for (18),

$$
I_{e}^{F V}=\sum_{k>k^{\prime}} \widetilde{\ln _{k^{\prime}}^{k}}\left[\operatorname{Im}\left(C_{e \tau}^{k} C_{e \tau}^{k^{\prime} *}\right)+\frac{m_{\mu}^{2}}{m_{\tau}^{2}} \operatorname{Im}\left(C_{e \mu}^{k} C_{e \mu}^{k^{\prime} *}\right)\right]
$$

one sees that the limit on $d_{e}$ from LFV depends more on $\tau \rightarrow e \gamma$ whose experimental upper bounds are still very far from providing useful constraints. Therefore this comparison is only possible in specific models and the class of models studied in this paper is particularly predictive in this respect as already discussed in section 5 .

\subsection{EDM in this class of models}

In the class of models discussed in the previous sections, the $C_{i j}^{k}$ are all related, so that $C_{e \mu}^{k}$ and $C_{e \tau}^{k}$ are generically $O(\epsilon) C_{\mu \tau}^{k}$. Therefore they are all constrained by the most significant limit, that from $\mathrm{BR}(\mu \rightarrow e \gamma)$ on $C_{e \mu}^{k}$. The potentially most important contribution to $d_{e}$ comes from the first term of $I_{e}^{F V}$ in (20) and is constrained by the decay $\tau \rightarrow e \gamma$. Replacing this term with eq. (13) one obtains, 
Lines of constant $\left|I_{e}^{F V}\right|^{u . b .}$
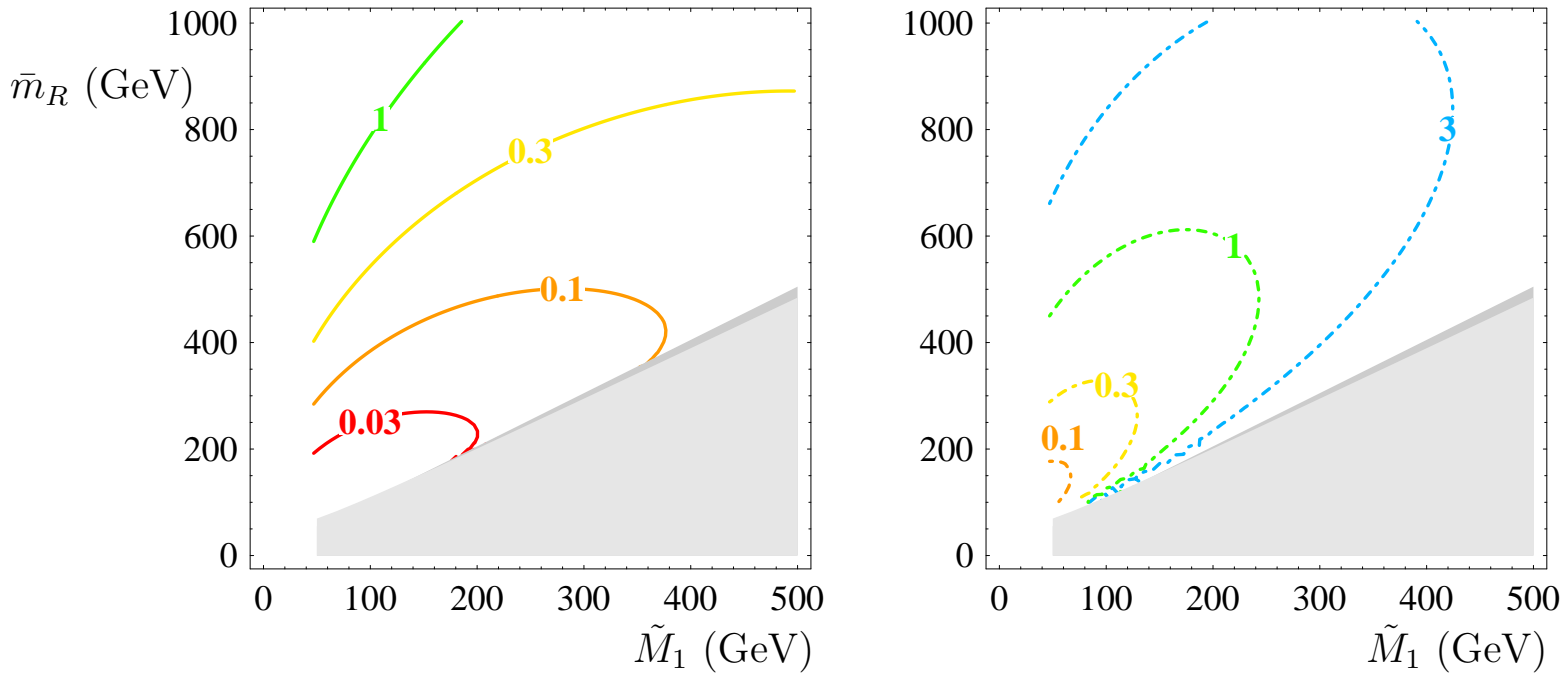

Figure 4: Upper limit on $\left|I_{e}^{F V}\right|$ from $d_{e} \lesssim 10^{-32}$ e cm with $\tan \beta=10, a_{0}=m_{0}+M_{1 / 2}$ (solid line) and $a_{0}=0$ (dotted line). For larger values of $\tan \beta$, multiply the upper bound for $(10 / \tan \beta)^{3}$.

$$
I_{e}^{F V}=\sum_{k>k^{\prime}} \widetilde{\ln _{k^{\prime}}^{k}} \ln \left(M_{P l} / M_{k}\right) \ln \left(M_{P l} / M_{k^{\prime}}\right) \frac{\epsilon^{2} M_{k} M_{k^{\prime}}}{M_{@}^{2}} \frac{\operatorname{Im}\left(h_{k 1}^{*} h_{k 3} h_{k^{\prime} 1} h_{k^{\prime} 3}^{*}\right)}{r_{k} r_{k}^{\prime} d_{@}^{2}}
$$

where the main contribution comes from $k=3, k^{\prime}=2$, the other ones being reduced by factors $O\left(M_{1} / M_{2}\right)$ and $O\left(M_{1} / M_{3}\right)$, respectively. The $\mu \rightarrow e \gamma$ decay giving already a limit on $M_{3}$, in principle, the experimental limits on the electron EDM and (21) yields a bound on $M_{2}$. However, as we now turn to discuss, the seesaw contribution to the lepton EDM comes out too small to give any relevant limit in the present class of models.

The maximum values of (21) correspond to $M_{2}=O\left(M_{3}\right)$ and are roughly given by

$$
I_{e}^{F V}=O\left(10^{-2}\right)\left(\frac{M_{3}}{M_{@}} \ln \frac{M_{P l}}{M_{3}}\right)^{2},
$$

which is maximum near the maximum value of $M_{3}$. As shown in section 5 for $\tan \beta \geq 10$ and sparticle masses below the $\mathrm{TeV}$, the present limit on $\mu \rightarrow e \gamma$ requires $M_{3} \lesssim M_{@}$, which implies $I_{e}^{F V} \lesssim 10^{-1}$. The planned improvement in $\mu \rightarrow e \gamma$ searches, at the level of $\operatorname{BR}(\mu \rightarrow e \gamma) \lesssim 10^{-14}$, would imply $M_{3} / M_{@} \lesssim 0.03$ in the 

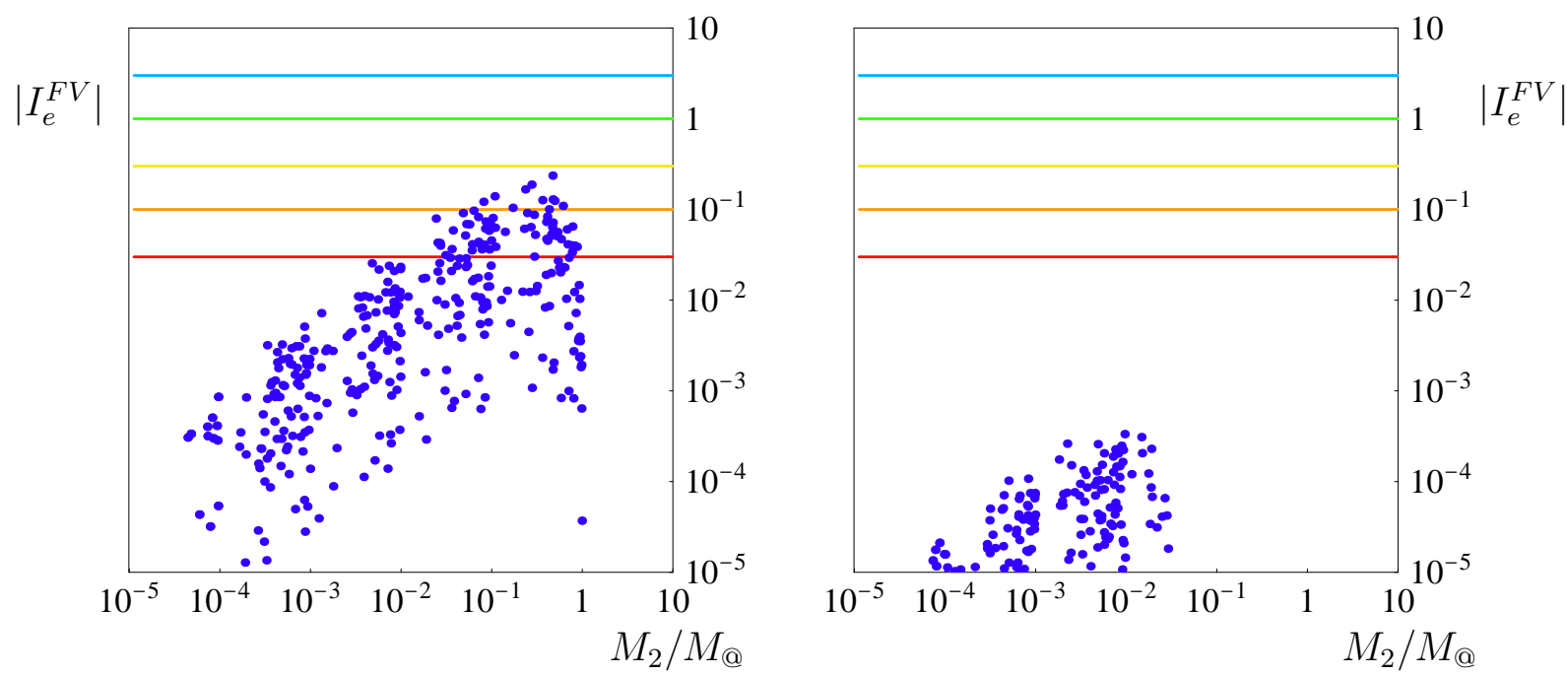

Figure 5: $\quad I_{e}^{F V}$ as a function of $M_{2} / M_{@}$ for $M_{3} / M_{@}=1$ and 0.03 respectively, with randomly generated models that fulfill the neutrino oscillation and leptogenesis experimental constraints with $M_{1}<10^{11} \mathrm{GeV}$.

same region of the MSSM parameter space and, correspondingly, $I_{e}^{F V} \lesssim 10^{-4}$, thus $d_{e} \lesssim 10^{-36} \mathrm{e} \mathrm{cm}$. Comparing these numbers with the plots in fig. 团 one concludes that, even with a bound of $10^{-32} \mathrm{e} \mathrm{cm}$ on $d_{e}$, this radiative contribution is too low to be detected.

The plots in fig. 5 show the dependence on $M_{2}$ for the models that satisfy all the experimental constraints of the previous sections. The vertical spreading of the points corresponds to the random overall phase. It is worth noting that the phases in $d_{e}$ and in $\varepsilon_{1}$ are uncorrelated. The former is generically proportional to $\operatorname{Im}\left(h_{31}^{*} h_{33} h_{21} h_{23}^{*}\right)$, while the latter rather depends on a combination of $\operatorname{Im}\left(h_{22} h_{12}^{*}+\right.$ $\left.h_{23} h_{13}^{*}\right)$ and $\operatorname{Im}\left(h_{32} h_{12}^{*}+h_{33} h_{13}^{*}\right)$, as previously discussed in section [4.

Notice also the predictions

$$
d_{\mu}=O\left(\epsilon^{-2} \frac{m_{\mu}}{m_{e}}\right) d_{e}=O(6000) d_{e}, \quad d_{\tau}=O\left(\epsilon^{-2} \frac{m_{\mu}^{2}}{m_{e} m_{\tau}}\right) d_{e}=O(400) d_{e}
$$

very different from the naive proportionality between lepton EDM and mass. Of course $d_{\mu}$ and $d_{\tau}$ are also predicted to be extremely small. 


\section{$7 \quad$ Decoupling the heavy neutrinos from $m_{@}$}

The fact that heavy Majorana masses end up pushed down by the experimental limits on LFV is due to the main characteristic of the models studied until now: as far as the heavy neutrinos commensurately contribute to the value of $m_{@}$, the only way to reduce the LFV from the Yukawa couplings is to decrease their masses. The alternative is to decouple one (or two) heavy neutrino from $m_{@}$. This is naturally done in the abelian flavour symmetry approach by the introduction of the so-called analytic zeros, imposed by the flavour symmetry and supersymmetry on the analytic superpotential. In the broken flavour symmetry phase, the zeros in the effective Yukawa interactions are filled by the diagonalisation of the Kahler metric which becomes $\epsilon$ dependent. Apparently, their values are diminished by powers of $\epsilon$, and so might be their contribution to LFV decays. Actually it turns out that, in the basis of the physical charged leptons, the couplings of the would-be decoupled state must be so much larger than those in the coupled case that the (partial) decoupling option is already experimentally excluded.

In this section, we try and make the expressions more transparent by omitting the $O(1)$ matrix elements defined and displayed in the previous ones. Correspondingly, we discuss only orders of magnitude and show the results in this form. More detailed calculations and definitions are presented in Appendix B, including a brief account of the transformations to the basis chosen here with diagonal $M_{R}$ and $Y_{\ell}$. It is also shown that the hierarchical structure, i.e., the $\epsilon$ dependence of $m_{\nu}$ as defined by (2), is stable under the necessary field redefinitions. From the discussion in section 3 we can already already take $\ell_{2}=\ell_{3}=\ell=\ell_{1}-1$, as in eq. (5), as well the relations in (6) and (77) below.

In order to decouple $\nu_{3}^{c}$ from $m_{@}$, we assume the charge $n_{3}$ to be large enough so that $n_{3}+\ell+h_{u}<0$. Since this charge imbalance cannot be compensated analytically, it implies that $Y_{\nu 3 i}=0(i=2,3)$ in the basis where the abelian flavour charge is diagonal. If one also chooses $n_{3}+\ell_{1}+h_{u}<0, \nu_{3}^{c}$ decouples from all light neutrinos, in this basis, and the model becomes of the so-called $3 \times 2$ type, which we skip here since it has received a lot of attention in the literature [48. As just explained, we are led to choose $\ell_{1}=\ell+1$ from (5). Hence $n_{3}+\ell_{1}+h_{u}=0$ and $Y_{\nu 31}=O(1)$. Then, from (17), we get

$$
M_{3}=O\left(\epsilon^{-2} M_{@}\right)=O\left(10^{16}\right) \mathrm{GeV},
$$

close to the GUT scale. Since $M_{k} / M_{3}=O\left(\epsilon^{2 n_{k}-2 n_{3}}\right)$, one has in the basis of the flavour charge eigenstates:

$$
Y_{\nu 31}=O(1), \quad Y_{\nu 3 j}=0 \quad(j=2,3), \quad Y_{\nu k j}=O\left(\epsilon^{\delta_{j 1}} \sqrt{\frac{M_{k}}{M_{@}}}\right)(k=1,2)
$$


In going to the physical basis where $M_{R}\left(Y_{\ell}\right)$ is diagonal, there are two steps: first to put the Kahler metric in canonical form, then to diagonalise $M_{R}$ ( $Y_{\ell}$, resp.). While in section 2 it has been remarked that this does not change the structure of the matrices in the models previously studied, here they fill the analytic zeros $Y_{\nu 3 j}=0(j=2,3)$. In this basis these couplings become (see Appendix B)

$$
\begin{aligned}
Y_{\nu 3 j} & \rightarrow \sum_{k=1,2} O\left(\frac{\sqrt{M_{3} M_{k}}}{M_{3}-M_{k}}\right) Y_{\nu k 3}+\epsilon \eta_{L 1 j} Y_{\nu 31} \\
& =O\left(\epsilon \frac{M_{2}}{M_{@}}\right)+O(\epsilon) \quad(i=2,3),
\end{aligned}
$$

where the $\mathrm{O}(1)$ coefficients come from a small rotation and a small Kahler vielbein, the first (second) terms are associated to the transformations of the right-handed neutrinos (left-handed charged leptons, resp.). Notice that these couplings are large, of $O(\epsilon)$, so large that the model is ruled out by LFV decays. Indeed, keeping only the leading $\nu_{2}^{c}$ and $\nu_{3}^{c}$ contributions, one gets:

$$
C_{\mu e}=\epsilon O\left(\frac{M_{2}}{M_{@}}\right) \ln \frac{M_{P l}}{M_{2}}+\epsilon\left(O(1)+O\left(\frac{M_{2}}{M_{@}}\right)\right) \ln \frac{M_{P l}}{\epsilon^{-2} M_{@}}=O(1) .
$$

This value is above its MSSM upper limit $\left|C_{\mu e}\right|^{\text {u.b. }}$, discussed in section 5 , even for quite heavy gauginos, $\tilde{M}_{1}=O(\mathrm{TeV})$. The only remaining possibility is to assume $M_{2}=O\left(M_{@}\right)$ and an (unnatural) cancellation between the various $O(1)$ terms that result. In this sense models with decoupling are (i) fixed up to $O(1)$ coefficients and (ii) basically already excluded by experiments. Remember that planned experiments on $\mu \rightarrow e \gamma$ will reduce $\left|C_{\mu e}\right|^{\text {u.b. }}$ by more than an order of magnitude.

Finally, let us just comment on the remaining possibility of decoupling both $\nu_{3}^{c}$ and $\nu_{2}^{c}$ from $m_{@}$, namely from both $\nu_{2}$ and $\nu_{3}$, so that one neutrino remains exactly massless while $\nu_{1}^{c}$ dominates in $m_{@}$. This could look interesting because, the tuning of $\rho$ examined in section 3 might become natural [29]. However, it is easy to check that this configuration leads to $m_{\odot} / m_{@}=O\left(\epsilon^{2}\right)$ and $\theta_{12}=O(\epsilon)$ requiring unnatural coefficients to fit the oscillation data. Of course the same constraints as in the case of only one decoupling from $m_{@}$ also apply here and require a tuning between the contributions from $\nu_{3}^{c}$ and $\nu_{2}^{c}$ to $C_{e \mu}$, which are both of $O(1)$. Altogether, this last case is even less attractive than the previous one.

\section{Conclusions}

In this paper we have emphasized the complementarity of some of the present and future experimental data for the determination of the Dirac and Majorana mass 
matrices of the supersymmetric seesaw models. For this purpose, we have chosen the simplest abelian flavour models, in which the structure of the Dirac and Majorana masses are fixed, up to $O(1)$ parameters with arbitrary phases, by only 8 parameters: the 6 lepton flavour charges, the small Froggatt-Nielsen parameter $\epsilon$ and the $B-L$ breaking scale. We have shown that the available data (basically the atmospheric and solar oscillation masses and angles, the baryon asymmetry in the universe and the $\mu \rightarrow e \gamma$ decay) are enough to fix the seesaw mass patterns to a large extent. These models predict the $\mu \rightarrow e \gamma$ decay to be within the reach of the planned searches. They also predict a very low contribution to $d_{e}$ which is bounded in this class of models by the limits on $\mu \rightarrow e \gamma$ alone. In other words, the lepton flavour abelian flavour models are consistent with experiments but only a few choices of the parameters are possible and basically all the selected models will be tested by the future experiments.

The models possessing all these properties are theoretically well-defined effective theories, the flavour symmetry is the simplest abelian one. The fact that a few experimental data of very different nature can complement each other to constrain so much the models is certainly non-trivial. Type I leptogenesis always requires one relatively light right-handed neutrino, but here it has to be at the upper side of the allowed region. It is well-known that the $\mu \rightarrow e \gamma$ experiments are testing the LFV effects in the slepton masses to the level of the radiative corrections, but here they immediately translate into a limit on a right handed neutrino mass eigenvalue and the whole series of LFV decays is predicted. Namely, the complementarity of the experimental constraints is disentangled into simple relations for the physical parameters.

Of course these models are special in many respects. They predict a hierarchical spectrum for the light neutrinos with $U_{e 3}$ close to its present bound, choosing one of the possible neutrino mass matrix patterns. They explains the large oscillation mixing angles by large mixings in the Dirac masses, favouring large LFV. The ratio between the solar and the atmospheric mass differences is directly related to a fundamental parameter, the scale of the flavour symmetry breaking, at the price of some tuning. Whenever all the right handed neutrinos couple to all left handed ones in the Yukawa matrix, they all contribute to the atmospheric neutrino oscillations. If one right state (not a mass eigenstate) decouples from the $\mu$ and the $\tau$ doublets, one Majorana mass eigenvalue can be quite large, close to the GUT scale. With the similar decoupling of another state, one gets the pattern corresponding to the dominance of the atmospheric oscillations by the lightest right handed neutrino, but $m_{\odot} / m_{@}$ and $\theta_{12}$ come out too small.

Thanks to supersymmetry, in the class of models examined here all these situations are naturally realized in the sense that they are ensured by the choice of the arbitrary charges. The results are quite independent of the various $O(1)$ free parameters, meaning that the conclusions are technically robust. The dependence on the sparticle masses is taken into account by analysing the results for a large range 
of values of the most important parameters (slepton and gaugino masses). On this basis, our main conclusions here are: (i) models involving decoupling through supersymmetric zeros are basically excluded and (ii) the whole class of models will be generically excluded in the near future, by the experimental bounds on the $\mu \rightarrow e \gamma$ decay - once the constraints from the present data on neutrino oscillations and leptogenesis are imposed.

General predictions of these models are the relations among the LFV charged lepton decay rates, and the very low upper bounds on the contributions to $d_{e}$. Actually the latter are generically expected [9, 10] if the seesaw couplings are the only source of CPV. Instead, if the couplings of the GUT heavy colour triplets related to proton decay are also introduced, one can achieve much larger values of $d_{e}$ [49, 10. In $S U(5)$ GUT, with the $\overline{5}$-plet and singlet charges fixed by the pure seesaw contributions, the 10-plet ones by the quark masses and mixings, the limits on $d_{e}$ are then predicted [50] up to uncertainties in $O(1)$ coefficients in the seesaw model, their phases, and the amounts of non-minimality in the GUT required to fit the data (fermion masses and mixings, proton decay). This analysis of $d_{e}$ in a GUT framework is also supported by the $O\left(m_{\odot} / m_{@}\right)$ value of $\epsilon$ obtained in the present paper, quite close indeed to the Cabibbo angle which is the natural choice in the quark sector.

Acknowledgements: The authors thank P. Di Bari for useful observations. The authors are grateful to the Dept. of Physics of "La Sapienza" University in Rome for the warm hospitality during the completion of this work. This work has been supported in part by the RTN European Program MRTN-CT-2004-503369.

\section{Appendix A}

The FC contribution of $A_{i} \equiv a_{i} Y_{\ell_{i}}$ has been calculated in refs. [9] and [10] at lowest order $^{5}$. In order to treat the various thresholds in a compact way, it is convenient to introduce the function defined as $\ln _{b}^{a}=\ln \left(M_{a} / M_{b}\right)$ where $a, b=1,2,3,4$ with the identification $M_{4}=M_{P l}$, as well as the matrices $N_{a}=Y_{\nu}^{\dagger} P_{a} Y_{\nu}$ with $P_{3}=\mathbb{I}$, $P_{2}=\operatorname{diag}(1,1,0), P_{1}=\operatorname{diag}(1,0,0)$, and it is understood that the Yukawas are taken at $M_{P l}$. Then [10]:

$$
\operatorname{Im}\left(a_{i}\right)=\frac{8}{(4 \pi)^{4}} a_{0} I_{i}^{F C} \quad, \quad I_{i}^{F C}=\sum_{a>b} \ln _{a}^{a+1} \ln _{b}^{b+1} \operatorname{Im}\left(N_{a} N_{b}\right)_{i i}
$$

\footnotetext{
${ }^{5}$ The coefficients in the papers of [9] numerically disagree with each other and with the result in ref. 10. The differences are irrelevant for the qualitative conclusions herein. Ref. [47] find, on the contrary, a smaller FC contribution.
} 
where the sum is over $a, b=1,2,3$.

The contribution from two insertions of FV slepton mass matrix elements obtained in [10, 47] reads:

$$
\begin{aligned}
\operatorname{Im}\left(\delta^{R R} m_{\ell} \delta^{L L}\right)_{i i} & =\frac{8}{(4 \pi)^{6}} m_{\ell_{i}} \frac{m_{\tau}^{2}}{m_{t}^{2}} \tan ^{2} \beta \frac{\left(6 m_{0}^{2}+2 a_{0}^{2}\right)\left(6 m_{0}^{2}+3 a_{0}^{2}\right)}{\bar{m}_{L}^{2} \bar{m}_{R}^{2}} I_{i}^{F V}, \\
I_{i}^{F V} & =\sum_{a>b} \ln _{a}^{a+1} \ln _{b}^{b+1}\left(\ln _{a}^{a+1}+\ln _{b}^{b+1}\right) \operatorname{Im}\left(N_{a} \frac{m_{\ell}^{2}}{m_{\tau}^{2}} N_{b}\right)_{i i}
\end{aligned}
$$

where $\bar{m}_{R}$ and $\bar{m}_{L}$ are average $\mathrm{R}$ and $\mathrm{L}$ slepton masses at low energy, and $Y_{\ell} \simeq$ $m_{\ell} \tan \beta / m_{t}$ has been substituted.

The FC contribution vanishes for $a_{0}=0$. On the other hand, for $a_{0} \sim m_{0}$, it turns out that the ratio $d_{i}^{F V} / d_{i}^{F C}$ is well approximated by the simple rule [10]

$$
\frac{d_{i}^{F V}}{d_{i}^{F C}} \approx 0.3 \frac{\ln _{2}^{P l}}{10}\left(\frac{\tan \beta}{10}\right)^{3}
$$

where $\mu$ has been fixed by electroweak symmetry breaking. Thus, the FV contribution is in general favoured by a factor $(\tan \beta / 10)^{3}$. A detailed comparison of these two contributions is in [10, together with limits on $I_{i}^{F V, F C}$ from the present and planned sensitivity to $d_{e}$ and $d_{\mu}$.

The above expressions for $I^{F V}$ and $I^{F C}$ can be rewritten in terms of the $C^{\prime}$ 's related to LFV as follows:

$$
\begin{aligned}
& I_{i}^{F V}=\sum_{a>b} I_{i}^{(a b) F V}, I_{i}^{(a b) F V}=\widetilde{\ln _{b}^{a}} \operatorname{Im}\left(C^{a} \frac{m_{\ell}^{2}}{m_{\tau}^{2}} C^{b}\right)_{i i}, \\
& \widetilde{\ln _{2}^{3}}=\ln _{2}^{3}, \quad \widetilde{\ln _{1}^{3}}=\ln _{1}^{3}-2 \frac{\ln _{2}^{3} \ln _{1}^{2}}{\ln _{1}^{P l}}, \quad \widetilde{\ln _{1}^{2}}=\ln _{1}^{2}\left(1-2 \frac{\ln _{3}^{P l} \ln _{2}^{3}}{\ln _{1}^{P l} \ln _{2}^{P l}}\right) .
\end{aligned}
$$

Hence, explicitely:

$$
\begin{aligned}
I_{1}^{F V} & =\sum_{a>b} \widetilde{\ln _{b}^{a}}\left[\operatorname{Im}\left(C_{13}^{a} C_{13}^{b *}\right)+\frac{m_{\mu}^{2}}{m_{\tau}^{2}} \operatorname{Im}\left(C_{12}^{a} C_{12}^{b *}\right)\right] \\
I_{2}^{F V} & =\sum_{a>b} \widetilde{\ln _{b}^{a}}\left[\operatorname{Im}\left(C_{23}^{a} C_{23}^{b *}\right)-\frac{m_{e}^{2}}{m_{\tau}^{2}} \operatorname{Im}\left(C_{12}^{a} C_{12}^{b *}\right)\right] \\
I_{3}^{F V} & =\sum_{a>b} \widetilde{\ln _{b}^{a}}\left[-\frac{m_{\mu}^{2}}{m_{\tau}^{2}} \operatorname{Im}\left(C_{23}^{a} C_{23}^{b *}\right)-\frac{m_{e}^{2}}{m_{\tau}^{2}} \operatorname{Im}\left(C_{13}^{a} C_{13}^{b *}\right)\right]
\end{aligned}
$$


On the other hand, for the FC contribution,

$$
I_{i}^{F C}=\sum_{a>b} I_{i}^{(a b) F C}, \quad I_{i}^{(a b) F C}=\frac{\ln _{b}^{a}}{\ln _{b}^{P l}} \operatorname{Im}\left(C^{a} C^{b}\right)_{i i}
$$

Explicitely:

$$
\begin{aligned}
I_{1}^{F C} & =\sum_{a>b} \frac{\ln _{b}^{a}}{\ln _{b}^{P l}}\left[\operatorname{Im}\left(C_{13}^{a} C_{13}^{b *}\right)+\operatorname{Im}\left(C_{12}^{a} C_{12}^{b *}\right)\right] \\
I_{2}^{F C} & =\sum_{a>b} \frac{\ln _{b}^{a}}{\ln _{b}^{P l}}\left[\operatorname{Im}\left(C_{23}^{a} C_{23}^{b *}\right)-\operatorname{Im}\left(C_{12}^{a} C_{12}^{b *}\right)\right] \\
I_{3}^{F C} & =\sum_{a>b} \frac{\ln _{b}^{a}}{\ln _{b}^{P l}}\left[-\operatorname{Im}\left(C_{23}^{a} C_{23}^{b *}\right)-\operatorname{Im}\left(C_{13}^{a} C_{13}^{b *}\right)\right]
\end{aligned}
$$

It is then manifest that $d_{e}$ is linked to $\tau \rightarrow e \gamma$ and $\mu \rightarrow e \gamma, d_{\mu}$ to $\tau \rightarrow \mu \gamma$ and $\mu \rightarrow e \gamma$, and $d_{\tau}$ to $\tau \rightarrow \mu \gamma$ and $\tau \rightarrow e \gamma$.

\section{Appendix B}

This appendix contains some details of the results discussed in section (7), namely, when a heavy neutrino partially decouples from the $\mu$ and the $\tau$. In the basis where $U(1)_{F}$ is diagonal this is naturally implemented by supersymmetry zeros in the $Y_{\nu}$ matrix. In the more convenient basis where $Y_{\ell}$ and $M_{R}$ are diagonal, these zeros are filled by: $i$ ) the vielbein redefinitions of both the light and heavy states [51], and ii) the unitary transformations - that remain free in the vielbein - to go into this basis,

in this order. In the framework of this paper, where generic $O(1)$ coefficients are free parameters and the calculations are carried on at lowest order, these tranformations commute. Therefore, one has to consider the effect of the tranformations:

$$
\begin{aligned}
M_{R} & \rightarrow V_{R}^{T} \xi_{R}^{T} M_{R} \xi_{R} V_{R}=\hat{M} \\
Y_{\ell}^{\dagger} Y_{\ell} & \rightarrow V_{L}^{\dagger} \xi_{L}^{\dagger} Y_{\ell}^{\dagger} Y_{\ell} \xi_{L} V_{L}=\hat{Y}_{\ell}^{2} \\
Y_{\nu} & \rightarrow V_{R}^{T} \xi_{R}^{T} Y_{\nu} \xi_{L} V_{L}=\hat{Y}_{\nu}^{2}
\end{aligned}
$$

where $\xi_{L, R}$ satisfy $\left(\xi \xi^{\dagger}\right)^{i \bar{j}}=K^{i \bar{j}}, K_{\bar{i} j}$ is the Kahler metric, $V_{R, L}$ are unitary, $\hat{M}$ and $\hat{Y}_{\ell}$ are diagonal. The metric is hermitean and invariant under $U(1)_{F}$, so that the transformation (40) can be generally written at the lowest order as follows, 


$$
\left(\xi_{R} V_{R}\right)_{i j}=\delta_{i j}+\eta_{R i j} \epsilon^{\left|n_{i}-n_{j}\right|}, \quad\left(\xi_{L} V_{L}\right)_{i j}=\delta_{i j}+\eta_{L i j} \epsilon^{\left|\ell_{i}-\ell_{j}\right|},
$$

where $\eta_{R, L}$ are $O(1)$ matrices whose hermitean parts bring the Kahler metric to the canonic form and whose anti-hermitean ones diagonalise $M_{R}$ and $Y_{\ell}$, respectively. Of course, the non-diagonal terms are not restricted by analyticity and there are no vanishing matrix elements, in general. When applied to $Y_{\nu}$ this transformation fills the possible analytic zeros already at the first order, as follows:

$$
Y_{\nu i j} \rightarrow \hat{Y}_{\nu i j} \approx Y_{\nu i j}+\sum_{k} Y_{\nu k j} \eta_{R k i} \epsilon^{\left|n_{i}-n_{k}\right|}+\sum_{k} Y_{\nu i k} \eta_{L k j} \epsilon^{\left|\ell_{j}-\ell_{k}\right|} .
$$

If $n_{i}$ and $\ell j$ are such that $Y_{\nu i j}$ is a supersymmetric zero, it becomes $\left(n_{i}, \ell j\right.$ decreasing with $i, j)$ :

$$
\hat{Y}_{\nu i j} \approx \sum_{k<i} \eta_{R k i} h_{k j} \epsilon^{2 n_{k}-n_{i}+\ell_{j}+h_{u}}+\sum_{k<j} \eta_{L k j} h_{i k} \epsilon^{2 \ell_{k}-\ell_{j}+n_{i}+h_{u}} .
$$

Notice that, for hierarchical $M_{R}$, the rotation angles of $V_{R}$ are:

$$
\left|\theta_{k i}\right| \approx\left|\frac{r_{i k}}{\sqrt{r_{i i} r_{k k}}} \frac{\sqrt{M_{i} M_{k}}}{M_{i}-M_{k}}\right|
$$

and the vielbein elements are of the same orders of magnitude.

A few comments are in order here. When the heavy neutrinos are integrated out to yield the effective $m_{\nu}$, the integration preserves analyticity as it does not involve the Kahler metric and the results could dependent only on the transformation $\xi_{L} V_{L}$ in (40). In the basis of the physical charged leptons one has at the lowest orders:

$$
\begin{aligned}
\hat{m}_{\nu i j} & =\left(V_{L}^{T} \xi_{L}^{T} m_{\nu} \xi_{L} V_{L}\right)_{i j} \\
& \approx m_{\nu i j}+\eta_{L k i} \epsilon^{\left|\ell_{i}-\ell_{k}\right|} m_{\nu k j}+m_{\nu i k} \eta_{L k j} \epsilon^{\left|\ell_{j}-\ell_{k}\right|}
\end{aligned}
$$

with $m_{\nu}$ given by eq. (21). It is easily checked that $\hat{m}_{\nu}$ has the same hierarchies in terms of $\epsilon$ as $m_{\nu}$, with the $O(1)$ coefficients reshuffled by $\xi_{L} V_{L}$. Instead, in the calculation of the effects of the RG evolution in LFV and CPV, the metric dependence is explicit in the results, even though they remain independent of $V_{R}$. For leptogenesis, the lepton asymmetry clearly depends on which is the lightest heavy neutrino and the whole transformation $\xi_{R} V_{R}$ is needed. Therefore it is convenient to work in the physical basis of the heavy neutrinos and to express all the quantities in this basis as done in section 7 . 


\section{References}

[1] P. Minkowski, Phys. Lett. B 67 (1977) 421; T. Yanagida in Proc. Workshop on Unified Theories, eds. O. Sawada and A. Sugamoto (Tsukuba, 1979); M. Gell-Mann, P. Ramond, and R. Slansky, in Supergravity, eds. D. Freedman et al., (North Holland 1980 Amsterdam) p. 315; R. N. Mohapatra and G. Senjanovic, Phys. Rev. Lett. 44, 912 (1980).

[2] J.A. Casas and A. Ibarra, Nucl. Phys. B 618 (2001) 171, hep-ph/0103065 S. Davidson and A. Ibarra, JHEP 0109 (2001) 013, hep-ph/0104076

[3] S. Lavignac, I. Masina and C.A. Savoy, Nucl. Phys. B 633 (2002) 139, hep-ph/0202086.

[4] I. Masina, Proceedings of SUSY 02, published in 'Supersymmetry and unification of fundamental interactions' vol.1 331 Hamburg (2002), hep-ph/0210125.

[5] G.C. Branco, R. Gonzalez Felipe, F.R. Joaquim, I. Masina, M.N. Rebelo, C.A. Savoy, Phys. Rev. D 67 (2003) 073025, hep-ph/0211001.

[6] S. Pascoli, S.T. Petcov, W. Rodejohann, Phys. Rev. D 68 (2003) 093007, hep-ph/0302054

[7] M. Fukugita and T. Yanagida, Phys. Lett. B 174 (1986) 45.

[8] F. Borzumati and A. Masiero, Phys. Rev. Lett. 57 (1986) 961.

[9] J. Ellis, J. Hisano, S. Lola and M. Raidal, Nucl. Phys. B 621 (2002) 208, hep-ph/0109125 J. Ellis, J. Hisano, M. Raidal and Y. Shimizu, Phys. Lett. B 528 (2002) 86, hep-ph/0111324.

[10] I. Masina, Nucl. Phys. B 671 (2003) 432, hep-ph/0304299

[11] S.T. Petcov, Sov. J. Nucl. Phys. 25 (1977) 340.

[12] M.L. Brooks et al. [MEGA Collaboration], Phys. Rev. Lett. 83 (1999) 1521, hep-ex/9905013.

[13] E.D. Commins, S.B. Ross, D. Demille, B.C. Regan, Phys. Rev. A 50 (1994) 2960;

B.C. Regan et al., Phys. Rev. Lett 88 (2002) 071805.

[14] C.D. Froggatt and H.B. Nielsen, Nucl. Phys. B 147 (1979) 277.

[15] N. Irges, S. Lavignac and P. Ramond, Phys. Rev. D 58 (1998) 035003, hep-ph/9802334 J.K. Elwood, N. Irges and P. Ramond, Phys. Rev. Lett. 81 (1998) 5064,hep-ph/9807228.

[16] F. Vissani, JHEP 9811 (1998) 025, hep-ph/9810435

[17] J. Sato and T. Yanagida, Phys. Lett. B 493 (2000) 356, hep-ph/0009205.

[18] G. Altarelli, F. Feruglio and I. Masina, JHEP 0301 (2003) 035, hep-ph/0210342. See also: R.N. Mohapatra, hep-ph/0211252 hep-ph/0306016 A.Y. Smirnov, hep-ph/0311259 
[19] W. Buchmuller and T. Yanagida, Phys. Lett. B 445 (1999) 399, hep-ph/9810308.

[20] W. Buchmuller, D. Delepine and F. Vissani, Phys. Lett. B 459 (1999) 171, hep-ph/9904219.

[21] J. Sato and K. Tobe, Phys. Rev. D 63 (2001) 116010, hep-ph/0012333.

[22] T. Blazek and S.F. King, Phys. Lett. B 518 (2001) 109, hep-ph/0105005.

[23] S. Lavignac, I. Masina and C.A. Savoy, Phys. Lett. B 520 (2001) 269, hep-ph/0106245.

[24] L.M. Barkov et al., proposal for an experiment at PSI, http://meg.web.psi.ch.

[25] J. Aysto et al.,Report of the Stopped Muons Working Group for the ECFA-CERN Study on Neutrino Factory and Muon Storage Rings at CERN, hep-ph/0109217; J.J. Hudson et al., Phys. Rev. Lett. 89 (2002) 023003, hep-ex/0202014 Y.K. Semertzidis, in Proceedings of the SIGHAD03 Pisa workshop, hep-ex/0401016.

[26] F.J.M. Farley et al, Phys. Rev. Lett. 93 (2004) 052001, hep-ex/0307006

[27] E. Dudas, S. Pokorski and C.A. Savoy, Phys. Lett. B 369 (1996) 255, hep-ph/9509410

E. Dudas, C. Grojean, S. Pokorski and C.A. Savoy, Nucl. Phys. B 481 (1996) 85, hep-ph/9606383 S.A. Abel and G. Servant, Nucl. Phys. B 611 (2001) 43, hep-ph/0105262 G.G. Ross and O. Vives, Phys. Rev. D 67 (2003) 095013, hep-ph/0211279

[28] See M.C. Gonzalez-Garcia, hep-ph/0410030, and references therein.

[29] S.F. King, Nucl. Phys. B 562 (1999) 57, hep-ph/9904210 Nucl. Phys. B 576 (2000) 85, hep-ph/9912492.

[30] W. Buchmuller, P. Di Bari and M. Plumacher, hep-ph/0401240 New J. Phys. 6 (2004) 105, hep-ph/0406014.

[31] B.A. Campbell, S. Davidson and K.A. Olive, Nucl. Phys. B 399 (1993) 111, hep-ph/9302223 L. Covi, E. Roulet and F. Vissani, Phys. Lett. B 384 (1996) 169, hep-ph/9605319 M. Plumacher, Nucl. Phys. B 530 (1998) 207, hep-ph/9704231.

[32] G.F. Giudice, A. Notari, M. Raidal, A. Riotto and A. Strumia, Nucl. Phys. B 685 (2004) 89, hep-ph/0310123.

[33] P. Di Bari, hep-ph/0406115.

[34] A. Pilaftsis, Phys. Rev. D 56 (1997) 5431, hep-ph/9707235; T. Hambye, Nucl. Phys. B 633 (2002) 171, hep-ph/0111089 J. Ellis, M. Raidal and T. Yanagida, Phys. Lett. B 546 (2002) 228, hep-ph/0206300 A. Pilaftsis and T.E. Underwood, Nucl. Phys. B 692 (2004) 303, hep-ph/0309342.

[35] M. Raidal, A. Strumia and K. Turzynski, hep-ph/0408015. See also: G.F. Giudice, A. Notari, M. Raidal, A. Riotto, A. Strumia, Nucl. Phys. B 685 (2004) 89, hep-ph/0310123 T. Hambye, Y. Lin, A. Notari, M. Papucci and A. Strumia, Nucl. Phys. B 695 (2004) 169, hep-ph/0312203 
[36] M. Tegmark et al., Phys. Rev. D 69 (2004) 103501.

[37] M. Boltz, W. Buchmuller and M. Plumacher, Phys. Lett. B 443 (1998) 209.

[38] M. Kawasaki, K. Kohri and T. Moroi, Phys. Rev. D 63 (2001) 103502, hep-ph/0012279.

[39] S. Davidson and A. Ibarra, Phys. Lett. B 535 (2002) 25; S. Davidson, JHEP 0303 (2003) 037, hep-ph/0302075.

[40] F. Gabbiani, E. Gabrielli, A. Masiero and L. Silvestrini, Nucl. Phys. B 477 (1996) 321 , hep-ph/9604387.

[41] J. Hisano, T. Moroi, K. Tobe, M. Yamaguchi and T. Yanagida, Phys. Lett. B 357, 579 (1995), hep-ph/9501407; J. Hisano and D. Nomura, Phys. Rev. D 59, 116005 (1999), hep-ph/9810479.

[42] I. Masina and C.A. Savoy, Nucl. Phys. B 661 (2003) 365, hep-ph/0211283.

[43] See, for instance: J. Sato, K. Tobe and T. Yanagida, Phys. Lett. B 498 (2001) 189, hep-ph/0010348 J. Hisano and K. Tobe, Phys. Lett. B 510 (2001) 197, hep-ph/0102315 J. Ellis, M. Raidal, Nucl. Phys. B 643 (2002) 229, hep-ph/0206174. A. Masiero, S.K. Vempati, O. Vives, Nucl. Phys. B 649 (2003) 189, hep-ph/0209303, T. Blazek and S.F. King, Nucl. Phys. B 662 (2003) 359, hep-ph/0211368: M. Ciuchini, A. Masiero, L. Silvestrini, S.K. Vempati, O. Vives, Phys. Rev. Lett. 92 (2004) 071801, hep-ph/0307191; S.M. Barr, Phys. Lett. B 578 (2004) 394, hep-ph/0307372, A. Masiero, S. Profumo, S.K. Vempati, C.E. Yaguna, JHEP 0403 (2004) 046, hep-ph/0401138 A. Masiero, S.K. Vempati, O. Vives, New J.Phys. 6 (2004) 202, hep-ph/0407325.

[44] For the connection with $(g-2)_{\mu}$ see e.g.: J. Ellis, M.E. Gomez, G.K. Leontaris, S. Lola and D.V. Nanopoulos, Eur. Phys. J. C14 (2000) 319; J. Hisano and K. Tobe, Phys. Lett. B 510 (2001) 197, hep-ph/0102315.

[45] T. Ibrahim and P. Nath, Phys. Rev. D 57 (1998) 478, 019901; Phys. Rev. D 58 (1998) 111301, Errata-ibid. D 60 (1999) 099902; M. Brhlik, G.J. Good, G.L. Kane, Phys. Rev. D 59 (1999) 115004, hep-ph/9810457; S. Pokorski, J. Rosiek and C.A. Savoy, Nucl. Phys. B 570 (2000) 81, hep-ph/9906206 S. Abel, S. Khalil and O. Lebedev, Nucl. Phys. B 606 (2001) 151, hep-ph/0103320 T. Ibrahim and P. Nath, Phys. Rev. D 64 (2001) 093002, hep-ph/0105025.

[46] A. Romanino and A. Strumia, Nucl. Phys. B 622 (2002) 73 hep-ph/0108275.

[47] Y. Farzan and M.E. Peskin, Phys.Rev. D70 (2004) 095001, hep-ph/0405214.

[48] R. Kuchimanchi, R.N. Mohapatra, Phys. Rev. D66 (2002) 051301, hep-ph/0207110, P.H. Frampton, S.L. Glashow, T. Yanagida, Phys. Lett. B 548 (2002) 119, hep-ph/0208157 G.C. Branco, R. Gonzalez Felipe, F.R. Joaquim, T. Yanagida, Phys. Lett. B 562 (2003) 265, hep-ph/0212341; B. Dutta, R.N. Mohapatra, Phys. Rev. D 68 (2003) 113008, hep-ph/0307163. 
[49] R. Barbieri and L.J. Hall, Phys. Lett. B 338 (1994) 212; R. Barbieri, L.J. Hall and A. Strumia, Nucl. Phys. B 445 (1995) 219, hep-ph/9501334.

[50] I. Masina and C.A. Savoy, Phys. Lett. B 579 (2004) 99, hep-ph/0309067.

[51] M. Leurer, Y. Nir, N. Seiberg, Nucl. Phys. B 420 (1994) 468, hep-ph/9310320. 